\title{
Immunogold labels: cell-surface markers in atomic force microscopy
}

\author{
Constant A.J. Putman ${ }^{\text {a }}$, Bart G. de Grooth ${ }^{\text {a }}$, Paul K. Hansma ${ }^{\text {b }}$, Niek F. van Hulst ${ }^{\text {a }}$ \\ and Jan Greve ${ }^{\text {a }}$ \\ a Department of Applied Physics, University of Twente, P.O. Box 217, 7500 AE Enschede, The Netherlands \\ ${ }^{b}$ Department of Physics, University of California, Santa Barbara, California 93106, USA
}

Received 1 July 1992

\begin{abstract}
The feasibility of using immunogold labels as cell-surface markers in atomic force microscopy is shown in this paper. The atomic force microscope (AFM) was used to image the surface of immunogold-labeled human lymphocytes. The lymphocytes were isolated from whole blood and labeled by an indirect immunolabeling method using the monoclonal antibody anti-CD3 and a secondary antibody (Goat-anti-Mouse) linked to $\mathbf{3 0} \mathrm{nm}$ colloidal gold particles. Some of the samples were enhanced by silver deposition onto the gold particles. The AFM images reveal the colloidal gold particles on the cell surface, with and without silver enhancement. Individual immunogold (-silver) particles are clearly resolved from the cell surface thus determining the location of antigens. The $30 \mathrm{~nm}$ gold particles appear in the AFM images having an average size of about 80 $\mathrm{nm}$ due to convolution between gold particle and AFM tip.
\end{abstract}

\section{Introduction}

A new type of high-resolution microscope was introduced by Binnig, Quate and Gerber in 1986 [1]. The atomic force microscope (AFM) is capable of high-resolution topographic imaging of sample surfaces. A sharp stylus is mounted on a cantilever with a low spring constant. The sample, which is mounted on a piezo electric $x y z$-translator, is raster-scanned beneath the stylus. Forces acting between atoms at the sample surface and atoms at the tip of the stylus will deflect the weak cantilever. Measuring the displacements of the tip and displaying them in a two-dimensional matrix gives a topographic representation of the sample surface.

Since its introduction, the AFM has been used to image a wide variety of samples, some of them revealing single atoms [2,3]. Biological samples form one of the major classes of samples which have been investigated. Red and white blood cells [4,5], DNA molecules [6-9], gap junctions [10], K562-cells (tumor cells) [11] and chromosomes
[11-13] are only a small part of the biological objects which have been imaged with the AFM. For a recent review on the application of the AFM on biological samples we refer to the paper by Hoh and Hansma [14]. A major advantage of the AFM is that the specimen requires no coating and the AFM can be operated under ambient conditions: no ultra-high vacuum is needed. Furthermore, Drake et al. [15] showed that the AFM can be successfully operated in an aqueous environment, thus enabling high-resolution imaging of biological objects and processes in a physiological environment.

One of the problems of imaging biological samples, e.g. cells, with the AFM is the lack of specificity; one cannot discriminate a specific membrane constituent from any other type. As in electron and light microscopy, it would be desirable to have a label that can be recognized with the AFM. The label to be recognized should have a certain physical morphology. Colloidal gold is a well known label, both in electron [16-19] and in light microscopy [20-24]. Conjugated to antibod- 
ies they form immunogold labels, which can be used to localize and quantify specific proteins on biological specimens.

In this paper we report the first successful attempt to detect cell-surface antigens on human lymphocytes using immunogold labels with the atomic force microscope. On some of the samples the silver enhancement technique, a photo-chemical reaction [25,26], was employed to increase the size of the gold particles $(30 \mathrm{~nm})$ by the deposition of silver on the particles. The images show the colloidal gold, with and without silver enhancement. Individual immunogold (-silver) particles are clearly resolved from the cell surface thus determining the locations of antigens.

\section{Materials and methods}

\subsection{Lymphocytes}

Human blood was obtained by venipuncture from a donor using heparine as anticoagulant. Lymphocytes were isolated according to the Percoll density gradient method. On these cells an indirect immunolabeling was performed. At the first stage, the cells were incubated with the monoclonal antibody anti-CD3 (Becton Dickinson, San Jose, CA., USA) for $30 \mathrm{~min}$ at $4^{\circ} \mathrm{C}$ in PBS containing $1 \% \mathrm{BSA}$ and $0.005 \% \mathrm{NaN}_{3}$. After washing two times, the cells were then incubated with a secondary antibody (Goat-antiMouse) linked to $30 \mathrm{~nm}$ gold particles (Janssen Pharmaceutica, Beerse, Belgium) for $60 \mathrm{~min}$ at room temperature. After two more washing steps cytospin slides were prepared. These were then fixed in buffered formaldehyde acetone. On some of the slides the silver enhancement method was performed according to the manufacturers instructions (intenSE M-kit, Janssen Pharmaceutica, Beerse, Belgium) for $10 \mathrm{~min}$. The samples were stored in the freezer. Before imaging, the samples were air-dried at room temperature for $30 \mathrm{~min}$ to remove the condensed water.

\subsection{The atomic force microscope}

The AFM used in this study is the Nanoscope II (Digital Instruments, Santa Barbara, CA, USA).

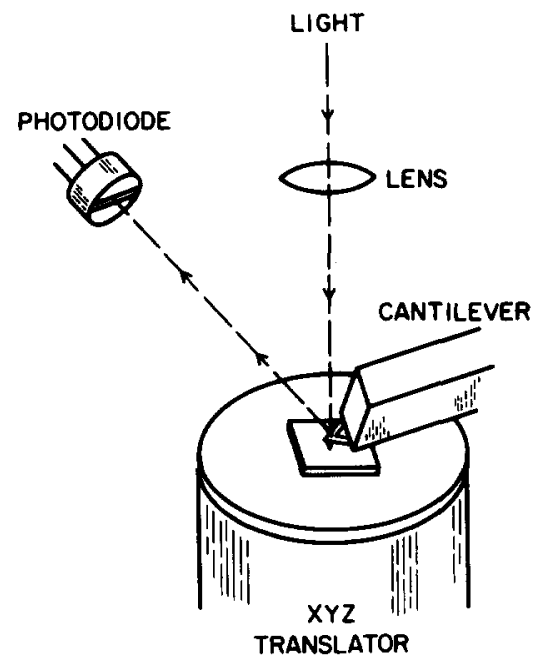

Fig. 1. Schematic diagram of the atomic force microscope. Laser light from a laser diode is focused on a cantilever which reflects it towards a segmented photodiode. The photodiode senses the deflection of the reflected beam and thus the deflection of the cantilever. In operation a feedback loop controls the position of the cantilever and hence the force applied to the sample. This is accomplished by moving the sample up and down with the translator as it is scanned underneath the cantilever tip.

The sample, glued to a small steel disc, is placed on a magnet mounted on a piezo electric $x y z$ translator (fig. 1). The sample is raster-scanned below a microfabricated cantilever with integrated pyramidal tip [27]. While scanning, surface features cause deflections of the cantilever. The displacements of the cantilever are detected using the optical beam deflection method $[28,29]$. A laser beam from a laser diode is focused on the reflective backside of the cantilever. The reflected beam is detected by a 2 -segment photodiode. A deflection of the cantilever results in a shift of the laser spot at the photodiode and generates a difference signal which is proportional to the displacement of the tip. This difference signal is used in a feedback loop to keep the cantilever deflection constant by applying a varying voltage to the $z$-electrode of the $x y z$-translator.

The images presented here were obtained using two modes of imaging. In the height mode the voltage applied to the $z$-electrode is plotted as function of the spatial position. The feedback 
loop, however, is not capable of compensating for fast variations in surface topography. In the error signal mode [30], the variations in difference signal which are not compensated by the feedback loop are plotted as function of the spatial position. In the height mode light features correspond to higher-lying objects and dark regions to lower-lying parts. Since in the error signal mode the edges of surface features are enhanced due to the compensation by the feedback loop (high-pass filtering), light corresponds to the up slope and dark to the down slope of a surface feature.

The images were recorded in air at a scan rate of 8.7 lines per second resulting in an imaging time of 46 seconds per image. The maximum scan area of the $x y z$-translator used was $83 \times 83 \mu \mathrm{m}^{2}$. We used $200 \mu \mathrm{m}$ long $\mathrm{Si}_{3} \mathrm{~N}_{4}$ cantilevers with a spring constant of $0.06 \mathrm{~N} / \mathrm{m}$. The applied force has typically a value of $10 \mathrm{nN}$.

\section{Results}

The cells labeled with immunogold and silverenhanced afterwards were imaged first. In fig. 2 the results of these measurements are shown. In fig. 2a a CD3-positive T-lymphocyte is shown. This picture was taken in the error signal mode resulting in a shaded-like appearance of the cell. The lines at the upper edge of the cell are artefacts due to the near-oscillation operation of the feedback loop. An almost uniform distribution of the immunogold-silver particles is visible. Furthermore, there is a certain variation in the sizes
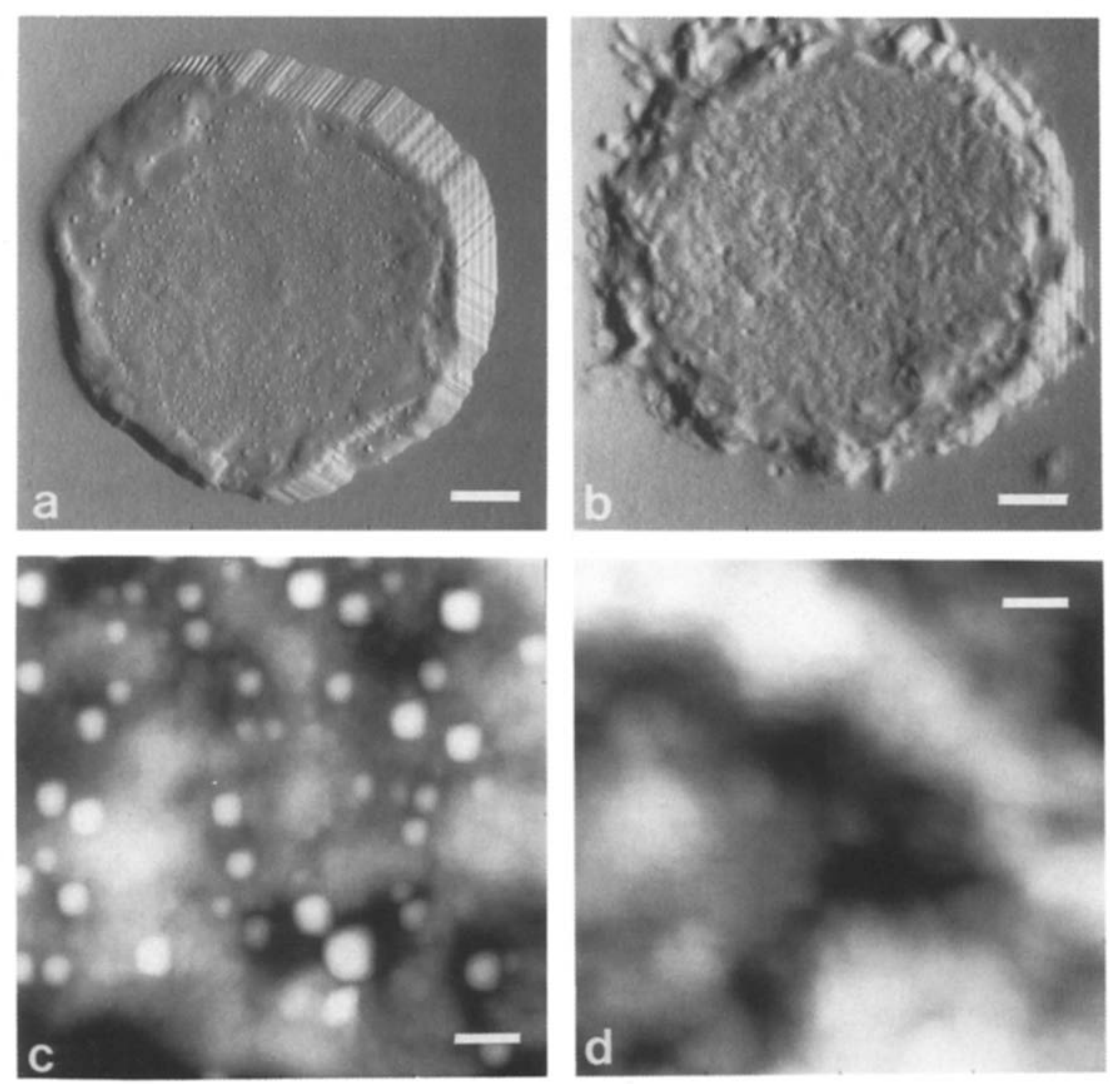

Fig. 2. AFM images of lymphocytes from an immunogold ( $30 \mathrm{~nm}$ )-labeled and silver-enhanced (10 min) sample. (a) CD3-positive lymphocyte, bar: $2 \mu \mathrm{m}$; (b) CD3-negative lymphocyte, bar: $2 \mu \mathrm{m}$; (c) detail of a CD3-positive lymphocyte, bar: $0.25 \mu \mathrm{m}$; (d) detail of a CD3-negative lymphocyte, bar: $0.25 \mu \mathrm{m}$. Images (a) and (b): error signal mode; and images (c) and (d): height mode. 
of the particles. This is more clearly visible in fig. $2 \mathrm{c}$ where a smaller area of a labeled lymphocyte is shown. The diameters of the particles vary over a range from 90 to $180 \mathrm{~nm}$. In fig. $2 \mathrm{~b}$ and $2 \mathrm{~d}$ the surface of a CD3-negative cell, on the same sample, is shown. This is either a B-lymphocyte or a NK-cell.

After detecting the cell-surface antigens using the technique of immunogold labeling and silver enhancement, samples of lymphocytes were taken that only carried the bare $30 \mathrm{~nm}$ immunogold particles. In fig. 3 AFM images of a CD3-positive ((a) and (c)) and a CD3-negative cell ((b) and (d)) are shown at different magnifications. Fig. 3c gives a detail of the cell shown in fig. 3a. The elongated shape of the gold particles is due to convolution between tip and particles. The aver- age diameter, taken along the horizontal direction of the particles, is 80 to $85 \mathrm{~nm}$.

\section{Discussion}

The results shown here clearly indicate the potential of immunogold as a marker of cellsurface receptors for use in atomic force microscopy. With the aid of the immunogold labels it is possible to determine the position of specific proteins on the cell surface. Figs. $2 a$ and $2 b$ show typical examples of cells which were imaged on the same sample. The ratio between cells showing many particles ("positive") and cells carrying a small number or no particles ("negative") is about $2: 1$. This is in agreement with the number for
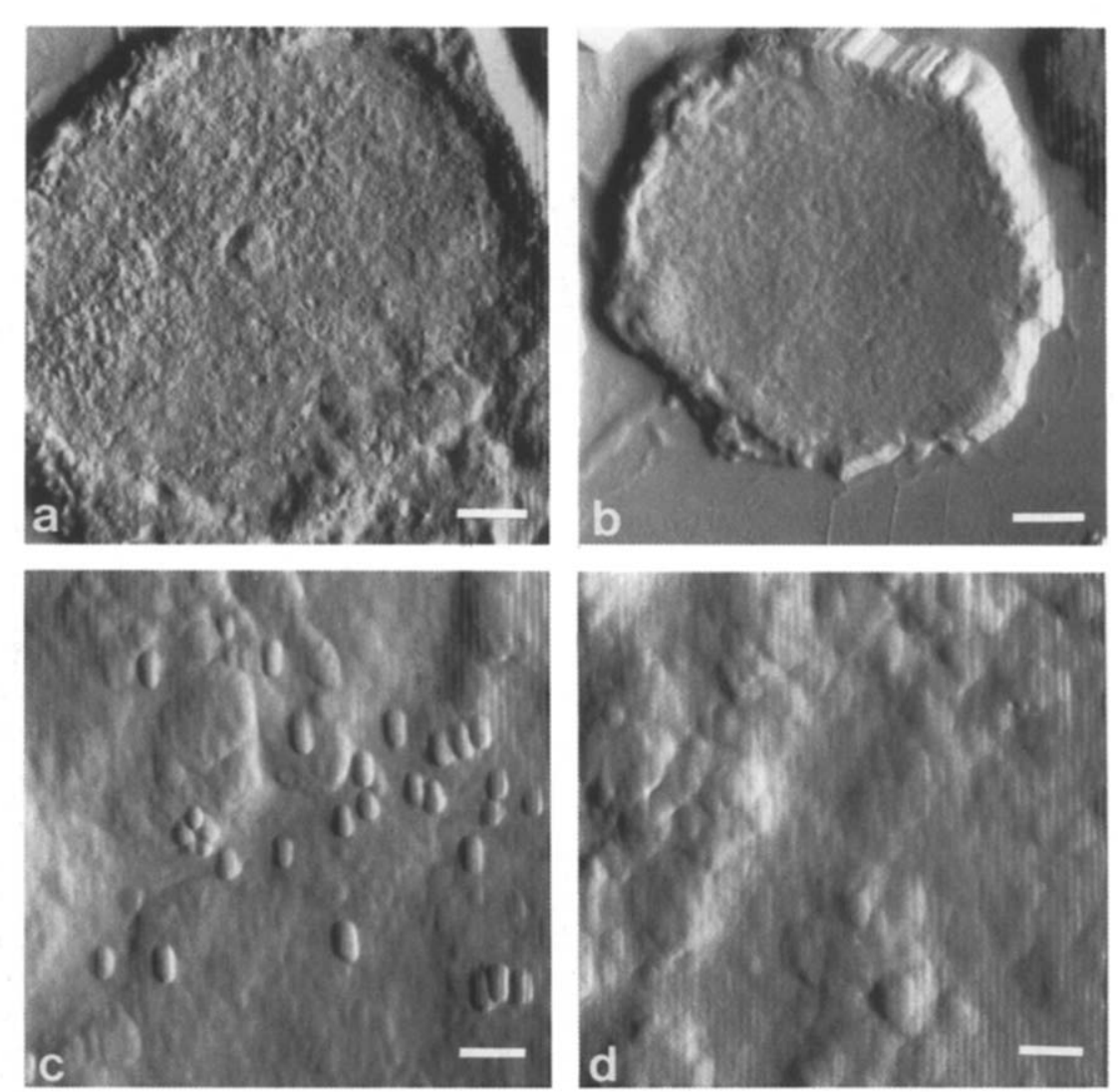

Fig. 3. AFM images of lymphocytes from an immunogold(30 nm)-labeled sample. (a) CD3-positive lymphocyte, bar: $2 \mu \mathrm{m}$; (b) CD3-negative lymphocyte, bar: $2 \mu \mathrm{m}$; (c) detail of the CD3-positive lymphocyte in (a) (upper left), bar: $0.25 \mu \mathrm{m}$; (d) detail of the CD3-negative lymphocyte in (b), bar: $0.25 \mu \mathrm{m}$. All images were taken in the error signal mode. 
lymphocytes with respect to the CD3-antigen obtained with fluorescence measurements. From fig. $2 \mathrm{c}$ it is clear that there is a large variation in imaged particle size. This indicates that the deposition of silver during the enhancement was nonuniform, as is known from experiment with the electron microscope [31]. The size increase of the colloidal gold due to the silver enhancement makes it easier to detect the labels, but as fig. 3 shows it is not neccessary. Smaller immunogold particles, e.g. 5 or $10 \mathrm{~nm}$, are more desirable than large particles, because the smaller particles have a higher labeling efficiency due to a reduced steric hindrance, which inhibits the bonding between the conjugated antibody (to the colloidal gold) and the cell-surface receptor. A combination of very small colloidal gold particles and silver enhancement is the best option for the detection of specific membrane proteins, as is used in electron microscopy [32].

Tip-particle convolution strongly influences the AFM images. The $30 \mathrm{~nm}$ particles appear in the AFM image (fig. 3c) having sizes of about $80 \mathrm{~nm}$ in the scan direction. This increase in imaged particle size can be explained by a convolution between tip and particle [8]. The tips we used typically have a radius of 30 to $40 \mathrm{~nm}$ and an included angle of $72^{\circ}$. As is shown in fig. 4, when imaging a sphere at a surface, e.g. a spherical gold-silver particle at a cell surface, the resulting trace is a convolution. The height of the object is measured correctly; the diameter is not. Apart

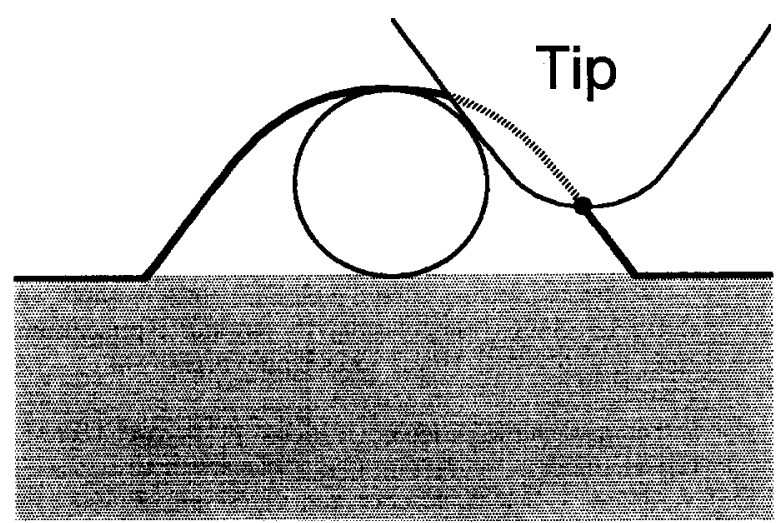

Fig. 4. Convolution of tip shape with spherical particle. The resulting trace gives a broadening of the particle. from the tip-object convolution, which is inherent to the AFM, a more serious problem involved in AFM operation is the variation in tip shapes. The elongated shape of the gold particles in fig. 3c indicates that the tip used was wedge-shaped. In fig. $2 c$ the particles appear to be more or less squares with rounded corners. Other AFM images show particles which are circular in shape. Due to limitations in the production process of the microfabricated cantilevers, there is a variation in tip shape, especially at the apex of the tip [27]. This variation in tip shape, however, does not limit the ability of the AFM to detect small immunogold particles. Although $30 \mathrm{~nm}$ particles increase to an imaged size of $80 \mathrm{~nm}$, the resolution of the AFM is not limited to $80 \mathrm{~nm}$. In fig. $3 \mathrm{c}$ smaller features are clearly visible. Furthermore, if the gold particles are packed close together with a center-to-center spacing of less than $80 \mathrm{~nm}$ (see fig. 3c, group of 5 particles left from center), individual particles can still be distinguished. With this type of convolution the resolution of the image is determined by the structure of both the tip and the sample.

An earlier attempt to image immunogoldlabeled cells (in an aqueous environment) with the AFM was made by Häberle et al. [33]. Red blood cells labeled with concanavalin A linked to $20 \mathrm{~nm}$ colloidal gold particles were imaged. No clear evidence was presented that indicated the presence of gold particles. On one of the cells a white bump was observed which might be associated with a cluster of colloidal gold particles.

The contrast mechanism in the contact AFM used stems from the hard-wall repulsion of overlapping electron clouds between atoms in tip and sample. The immunogold label has a distinctive morphology which makes it possible to discriminate between label and surrounding biological material. In the future we envision the development of an AFM-tip-sensitive label, meaning that the interaction between tip and label is different from the interaction between surrounding biological material and tip. A candidate label could be the existing ferromagnetic labels (available from SIGMA) in combination with a magnetic tip. With such a label protein complexes on cell membranes can be detected with high specificity. 


\section{Conclusions}

It was shown that immunogold particles of 30 $\mathrm{nm}$, with and without silver enhancement, can be detected on the cell membrane of human lymphocytes with the atomic force microscope. The results indicate that immunogold labels can be useful for structural studies of cell membranes and organelles with the atomic force microscope.

\section{Acknowledgements}

We thank Ine Segers for the preparation of the samples, Jason Cleveland for the graphics of fig. 4 and Jan Hoh for suggestions and discussion. This research was supported by the Netherlands Organization for Scientific Research NWO (C.A.J.P.) and the Office of Naval Research (P.K.H.).

\section{References}

[1] G. Binnig, C.F. Quate and C. Gerber, Phys. Rev. Lett. 56 (1986) 930.

[2] D. Rugar and P.K. Hansma, Phys. Today 43 (1990) 23.

[3] A. Engel, Annual Rev. Biophys. Biophys. Chem. 20 (1991) 79.

[4] S.A.C. Gould, B. Drake, C.B. Prater, A.L. Weisenhorn, S. Manne, H.G. Hansma, P.K. Hansma, J. Massie, M. Longmire, V. Elings, B. Dixon Northern, B. Muk rgee, C.M. Peterson, W. Stoeckenius, T.R. Albrecht and C.F. Quate, J. Vac. Sci. Technol. A 8 (1990) 369.

[5] H.J. Butt, E.K. Wolff, S.A.C. Gould, B. Dixon Northern, C.M. Peterson and P.K. Hansma, J. Struct. Biol. 105 (1991) 54.

[6] S.M. Lindsay, L.A. Nagahara, T. Thundat, U. Knipping, R.L. Rill, B. Drake, C.B. Prater, A.L. Weisenhorn, S.A.C. Gould and P.K. Hansma, J. Biomol. Struct. Dyn. 7 (1989) 279.

[7] A.L. Weisenhorn, H.E. Gaub, H.G. Hansma, R.L. Sinsheimer, G.L. Kelderman and P.K. Hansma, Scanning Microsc. 4 (1990) 511.

[8] C. Bustamante, J. Vesenka, C.L. Tang, W. Rees, M. Guthold and R. Keller, Biochemistry 31 (1992) 22.

[9] H.G. Hansma, J. Vesenka, C. Siegerist, G. Kelderman, H. Morrett, R.L. Sinsheimer, V. Elings, C. Bustamante and P.K. Hansma, Science 256 (1992) 1180.

[10] J.H. Hoh, R. Lal, S.A. John, J.P. Revel and M.F. Arnsdorf, Science 253 (1991) 1405.
[11] C.A.J. Putman, H.O. van der Werf, B.G. de Grooth, N.F. van Hulst, F.B. Segerink and J. Greve, Rev. Sci. Instrum. 63 (1992) 1914.

[12] B.G. de Grooth, G.A.J. Putman, K.O. van der Werf, N.F. van Hulst, G. van Oort and J. Greve, Proc. SPIE 1639 (1992) 205.

[13] W. Heckl, Thin Solid Films 210/211 (1992) 640.

[14] J.H. Hoh and P.K. Hansma, Trends Cell Biol. 2 (1992) 208.

[15] B. Drake, C.B. Prater, A.L. Weisenhorn, S.A.C. Gould, T.R. Albrecht, C.F. Quate, D.S. Cannell, H.G. Hansma and P.K. Hansma, Science 243 (1989) 1586.

[16] M. Horrisberger, Experientia 31 (1975) 1147.

[17] E. de Harven, R. Leung and H. Christensen, J. Cell. Biol. 99 (1984) 53.

[18] P. Walther and M. Muller, Detection of small (5-15 nm) gold labelled surface antigens using backscattered electrons, in: Science of Biological Specimen Preparation, Eds. M. Muller, R.P. Becker, A. Boyde and J.J. Wolosewick (SEM, Chicago, 1986) p. 195.

[19] D. Soligo, G. Lambertenghi-Deliliers and E. De Harven, Immunocytochemistry with backscattered electrons, in: Science of Biological Specimen Preparation, Eds. M. Muller, R.P. Becker, A. Boyde and J.J. Wolosewick (SEM, Chicago, 1986) p. 289.

[20] W.D. Geoghegan, J.J. Scillian and G.A. Ackerman, Immunol. Commun. 7 (1978) 1.

[21] M. de Waele, J. de Mey, M. Moeremans, M. de Brabander and B. van Camp, J. Histochem. Cytochem. 31 (1983) 376.

[22] M. de Brabander, G. Geuens, R. Nuydens, M. Moeremans and J. de Mey, Cytobios 43 (1985) 273.

[23] C.M. Hoefsmit, C. Korn, N. Blijleven and J.S. Ploem, J. Microscopy 143 (1986) 161.

[24] I. Cornelese-ten Velde, J. Bonnet, H.J. Tanke and J.S. Ploem, J. Microscopy 159 (1990) 1.

[25] G. Danscher and J.O.R. Nörgaard, J. Histochem. Cytochem. 31 (1983) 1394.

[26] C.S. Holgate, P. Jackson, P.N. Cowen and C. Bird, J. Histochem. Cytochem. 31 (1983) 938.

[27] T.R. Albrecht, S. Akamine, T.E Carver and C.F. Quate, J. Vac. Sci. Technol. A 8 (1990) 3386.

[28] G. Meyer and N.M. Amer, Appl. Phys. Lett. 53 (1988) 2400.

[29] C.A.J. Putman, B.G. de Grooth, N.F. van Hulst and J. Greve, J. Appl. Phys. 72 (1992) 6.

[30] C.A.J. Putman, H.O. van der Werf, B.G. de Grooth, J. Greve and P.K. Hansma, Proc. SPIE 1639 (1992) 198.

[31] L. Scopsi, Silver-enhanced colloidal gold method, in: Colloidal Gold: Principles, Methods, and Applications, Vol. I, Ed. M.A. Hayat (Academic Press, San Diego, 1989) p. 251.

[32] E. Namork and E.H. Heier, J. Electron Microsc. Tech. 11 (1989) 102.

[33] W. Häberle, J.K.H. Hörber and G. Binnig, J. Vac. Sci. Technol. B 9 (1991) 1210. 